\title{
Dieudonné Niangouna parle de Sony
}

Dieudonné Niangouna

\section{OpenEdition \\ Journals}

Édition électronique

URL : http://journals.openedition.org/coma/615

DOI : $10.4000 /$ coma.615

ISSN : 2275-1742

\section{Éditeur}

Institut des textes \& manuscrits modernes (ITEM)

\section{Référence électronique}

Dieudonné Niangouna, « Dieudonné Niangouna parle de Sony », Continents manuscrits [En ligne], 4 | 2015, mis en ligne le 26 janvier 2016, consulté le 16 avril 2021. URL : http://journals.openedition.org/ coma/615 ; DOl : https://doi.org/10.4000/coma.615

Ce document a été généré automatiquement le 16 avril 2021.

\section{(c) (i) ()}

Continents manuscrits - Génétique des textes littéraires - Afrique, Caraîbe, dispora est mis à disposition selon les termes de la licence Creative Commons Attribution - Pas d'Utilisation Commerciale - Pas de Modification 4.0 International. 


\title{
Dieudonné Niangouna parle de Sony
}

\author{
Dieudonné Niangouna
}

\section{Q : Quelle a été votre rencontre avec Sony?}

$\mathrm{R}$ : Sony, je le rencontre parce que d'abord papa avait une bibliothèque à la maison, on est dans un endroit où il $\mathrm{y}$ a plein de livres donc tu lis, tu sors de la bibliothèque de ton père, après tu commences à chercher d'autres bouquins. Il y a d'autres auteurs que je voulais connaître, qui n'étaient pas dans la bibliothèque de mon père. Et donc tu lis un bout d'africain, un bout de X, un bout de Y... et puis bon, de toutes les façons son nom était déjà connu, donc je ne rencontre pas d'abord l'écriture de Sony avant ce qui l'a précédé. C'est que, nous on était gamins, son nom était connu au Congo, rien que le nom était connu. Donc le nom, comme beaucoup de gens connaissent le nom de Tchicaya U Tam'si, même s'ils ne l'ont pas encore lu, Sony c'est pareil, le nom existait. Donc tout d'abord tu sais très bien qu'il y a un nom qui existe qui s'appelle Sony Labou Tansi. Puis, famille intello, donc facilement tu apprends que ce mec là il a écrit des bouquins, il écrit des livres et qu'il dirige une troupe de théâtre. Donc c'est beaucoup plus quand je vais chercher d'autres bouquins que je ne trouvais pas dans la bibliothèque de mon père, donc je vais chercher chez les petits revendeurs, tu lis un bout d'africain, un bout de japonais, qu'évidemment par curiosité tu tombes aussi sur Sony ou tu lis aussi Sony. Donc c'est une rencontre qui ne vient pas du fait qu'il ne m'est pas tombé dessus, il était déjà né avant moi, il existait, sa célébrité l'avait précédé, et du coup comme je me suis amusé à lire donc facilement, d'une certaine manière j'allais croiser un bouquin de Sony entre mes mains. Mais après, ça, c'est pas si spécial que ça d'être tombé sur un bouquin de Sony, parce qu'on est nombreux à être tombés dans les bouquins de Sony, et après c'est pas ça forcément qui fait qu'une personne croit en l'écriture de Sony, ou qu'il ait une affection envers ce qu'il raconte, ou qu'il soit un adepte ou un élève de Sony. Là c'est beaucoup plus des questions très très personnelles, de ce que tu défends, ce que tu aimes défendre, que ce soit dans l'écriture, que ce soit dans la vision du monde. Et moi je crois que ce qui m'intéressait quand je lisais des auteurs, depuis gamin même, depuis la bibliothèque de mon père, c'était deux choses toutes réunies. La première c'est la poésie d'un auteur, et la deuxième sa poétique. Ça a toujours été une chose comme ça qui m'a toujours intéressé. Un auteur qui disait des choses forcément intéressantes et qui n'avait pas 
pour moi une poésie de les dire ça ne m'intéressait pas vraiment. Par contre ce qu'ils disaient m'intéressait mais ce n'étaient pas des auteurs que je pouvais relire et relire et que je pouvais citer. Tout comme un auteur qui avait une belle langue, on pourrait le dire comme ça, ou qui aurait inventé la langue tout ce que tu veux, et qui n'a rien d'intéressant à raconter, ça ne m'intéressait pas non plus. Et donc du coup je crois que le choc s'opère quand évidemment je lis donc, la première œuvre de Sony que je lis c'est «La parenthèse de sang ». Je sais pas je devais avoir 10 ans ou 11 ans, je lis « la parenthèse de sang » ben voilà, c'était complètement très très vrai. Très très vrai parce que il y avait sa poésie, sa relation avec la langue, sa relation avec la parole, et puis il y avait tout à fait l'autre chose, qui est le plus intéressant, ou les deux sont intéressantes, qui est évidemment ce qu'il était en train de dire, ce qu'il était en train de pointer du doigt, ce qu'il était en train d'indexer et comment il était en train de vouloir à tout prix refabriquer la vie. Il avait complètement une espèce de passion incroyable à vouloir refabriquer la vie, à vouloir ré-inventer la vie, il s'en foutait que ce soit comme ça, ce qui l'intéressait c'est ce que lui rêve de bien, ce que lui pense de bien à cet endroit là, que ce soit au niveau des idéaux, que ce soit au niveau de la vie simplement comme ça, que ce soit au niveau des conceptions des choses. C'est qu'à première vue j'ai vu quelqu'un qui se mettait à donner une autre valeur même à la nomination des choses, c'est à dire on pouvait s'arrêter à dire «ben tiens il a salué quelqu'un ", mais lui ne s'arrêterait pas en disant que c'était quelqu'un. Il va ramener de la personnalité dans ce quelqu'un là, donc c'est comme si, oui, un truc comme ça assez gourmet, avec le sens qu'ont les mots, comme ils ont été tellement galvaudés, ils tellement été retournés tout ce que tu veux, si bien qu'ils sont rapidement devenus très très appauvris. Et donc du coup cette première relation c'est une espèce de fulgurance de redécouvrir les mots mais découvrir leur réel sens c'est à dire une espèce de valeur comme ça, qu'il donnait aux mots, une espèce d'identité, ça devenait comme des personnages, ça devenait comme des gueules les mots, parce que évidemment il les chargeait, il les remplissait de leur réelle mission. Donc du coup à partir de là, je lis Sony la première fois après quand j'essaie de lire autre chose, j'avais l'impression que l'autre là, pas que l'autre là ne savait pas écrire mais que les mots qu'il me disait là ce n'était pas les mots qu'il fallait pour qu'ils soient à leur place quoi. Donc du coup, c'est une rencontre assez forte comme ça, qui du coup rapidement va m'emmener à continuer à fouiller du Sony. Dans Brazzaville ça n'était pas facile d'en trouver même s'il était publié, même s'il avait une troupe de théâtre, trouver les bouquins de Sony c'était pas facile à l'époque. Jusqu'à maintenant c'est toujours pas facile au Congo. Il n'y en avait pas donc tu vas demander qui en a, quel grand l'a, quel étudiant, quel professeur l'a. Des fois par hasard, tu peux en trouver chez le petit revendeur, pas parce que il y en a beaucoup mais parce que quelqu'un avait faim il avait un bouquin chez lui il ne savait pas quelle importance ça a, il est parti au petit revendeur il a dit «Grand, donne moi 200 et je te vends un livre». Et toi tu passes là par hasard, ce jour là, tu le vois tu fais "ah", tu l'achètes. Donc là rapidement comme ça je me suis mis à chasser du Sony partout et à trouver et donc à 12 ans je vais lire "L'état honteux ». Là c'était une vraie vraie claque, cette histoire Martillimi Lopez, fils de Maman Nationale, qui était commandant de sa hernie, c'était incroyable, c'était autant jubilatoire, c'était autant ubuesque, en même temps destroy, mais d'un destroy incroyable mais en même temps très très sensé sur ce qu'il était en train de dire et surtout savoir comment le dire. C'est ce qui m'a beaucoup plu chez ce type là, c'est qu'il y a un problème, mais il ne s'arrête pas au problème qu'il a 
à dire. C'est qu'il va trouver une intelligence de le dire, mais cette intelligence là va être la sienne. Elle va être la sienne c'est à dire il va inventer son alphabet, il va inventer son langage pour qu'évidemment il ne puisse pas corrompre sa manière de penser, pour qu'il soit à même pour qu'il soit d'accord avec comment il pense il faut qu'il le formule avec sa manière de penser à lui. Sa manière de penser à lui veut dire quoi ? Faut qu'il invente sa poésie à lui, pour que évidemment... Moi c'est la plus belle chose que Sony m'a appris et c'est pas si compliqué que ça, la plus belle chose qu'il m'a appris c'est que, en lingala on dit «tu ne peux pas faire des plans sur la fête de quelqu'un d'autre " je sais pas comment le dire... Toi tu as acheté un bouquet de poisson à l'étouffé un Liboké, c'est ton poisson à toi, je dis « Ah Marotte a acheté des poissons alors qu'est ce que je fais? » Alors sans te demander « est-ce que je peux me couper un bout?» non non, je vais acheter un manioc mais sans te dire que j'ai du manioc... je fais un programme sur ta bouffe et c'est le propriétaire du chien qui lui coupe la queue. Tu ne peux pas prendre ta machette et couper la queue du chien du voisin en lui disant « parce que j'aime pas la queue !», ça n'a aucun sens. C'est à dire, c'est cette intelligence là réellement de comprendre que tu as pensé mais une fois de plus tu vas utiliser un langage pour te faire entendre, pour t'exprimer. Tu vas utiliser un langage, donc un certain nombre de canaux, signaux, tout ce que tu veux qui vont raconter vraiment ton langage. Mais si ces canaux là ne sont pas les tiens, ta pensée pourtant elle était tienne_ elle devient complètement esclave à l'intérieur d'un canal et $\mathrm{du}$ coup tu ne peux pas être à même de savoir défendre réellement ta pensée. Quand on dit « défendre » ce n'est pas question de défendre, il est question de savoir la raconter. Pas seulement pour la partager, pour être à même de savoir qu'elle est mienne. Il faut évidemment qu'elle pue tes odeurs à toi. Il faut évidemment qu'elle pue tes odeurs à toi. Il faut qu'elle ait la forme de comment tu as pensé cette chose là. Sinon ça ne marche pas, ça ne marche pas. Donc c'est cette réelle intelligence que j'ai eu rapidement qu'il m'a enseignée lui, à cet endroit de se dire « c'est à l'intérieur de son tronc humain, de sa moelle, qu'on fabrique, qu'on raconte sa pensée ». On ne raconte pas sa pensée avec le canal de l'autre ça ne marche pas. C'est comme cette femme, cette tantine là, elle sait faire des galettes, elle a lu la formule de comment faire les galettes tout le blablabla là, tu mélanges un bout de citron, un bout de levure ok. Mais par contre la main, c'est la main là qui va fabriquer les galettes, c'est la main de l'autre là. C'est pas que la galette ne sera pas belle, mais ça ne marchera pas, ce ne sera pas ses galettes à elle. Tu peux pas prendre la main de quelqu'un et dire «tu tournes comme ça...» non, non. C'est cette chose là qui est intéressante. Et c'est une réelle forme de responsabilité et d'intelligence à cet endroit-là. Donc moi, c'est à cet endroit que j'ai vu cette force de liberté, cette force d'autonomie, cette force de la recréation de soi. Il ne s'agit pas simplement d'avoir une pensée ou une idée, il s'agit de d'abord construire sa barque, la barque dans laquelle cette pensée s'auto-génère et à partir de laquelle elle parle, à partir de laquelle elle pense. Pour qu'évidemment ce ne soit pas une chose collée dans une autre. Comme disait ma grand-mère: «il ne faut pas que ce soit un timbre sur une enveloppe ", c'est l'expression de ma grandmère... Ça veut dire quoi ? Le timbre on l'a posé sur l'enveloppe mais le timbre n'est pas au courant de ce qu'on a dit dans la lettre, peut-être à l'intérieur de la lettre c'est un avis de mort, c'est peut-être une menace, mais le timbre sur l'enveloppe il fait comme ça «Welcome! ». Le timbre sur l'enveloppe c'est absolument le truc le plus dégueulasse. Et donc il m'a appris réellement cette chose-là, qu'évidemment la parole c'est un tout. Et elle n'est pas simple, l'écriture est un tout, l'acte est un tout. Il n'est 
pas ou elle n'est pas simplement forte parce que l'idée qu'il a dit est belle, non, non, non. Et l'idée, et sa forme et son machin doit être une seule chose, doit être complètement une seule chose, pour qu'évidemment on ne puisse pas séparer du gombo son gluant. Ce qui fait le gombo c'est son gluant. Il n'y a pas le gombo puis le gluant. Le gombo c'est le gluant, le gluant c'est le gombo. C'est une seule chose. C'est à cet endroit que ça l'est réellement, qu'évidemment il n'y a pas tricherie de sens. La chose qui parle ne peut pas se tromper. Elle ne peut pas être trompée par la forme, elle ne peut pas être trompée par l'entendement parce qu'elle est une seule chose. Elle est complètement une seule chose. Et donc cette forme de re-fabrication de langage n'a pas évidemment un orgueil d'avoir un bon langage, n'a pas un orgueil d'avoir un mauvais langage, ça n'a évidemment que la prétention de la recherche à être elle-même.

\section{Quelle citation de Sony vous habite aujourd'hui?}

Toutes. Non mais toutes. Toutes celles que je connais. J'en ai pas de meilleure plus qu'une autre. Je vais commencer par "repousser à plus tard la mort de la vie " «forcer la vie à être »... Tout ce que tu veux c'est... c'est... «je ne suis pas à développer mais à prendre ou à laisser ", "l'Afrique n'est pas mal partie, l'Afrique n'est jamais partie " «l'être humain est trop beau pour qu'on le néglige » «je suis intentionnellement humain », j'en ai pas une que je peux dire la meilleure, «ça c'est celle qui m'habite ». Par exemple comment j'ouvre à chaque fois Mantsina depuis 10 ans, je dis toujours "sommes-nous sortis du monde Riforoni? ». C'est même pas une citation de Sony, c'est la première tirade de sa pièce "Antoine m'a vendu son destin », la première tirade du personnage d'Antoine qui est avec son garde du corps à côté _avec son ministre tout ce que tu veux_ ils font silence, ils se concentrent et il lui dit «sommes-nous sortis du monde Riforoni? » et Riforoni lui répond «nous en sommes sortis votre Altesse" "sommes nous les derniers du monde? Seules nos respirations nous écoutent, nous sommes seuls.» "Alors lis la déclaration que le peuple va lire ... ». Cette phrase là « Sommes-nous sortis... », ce n'est pas une citation, ça n'a rien d'une citation. C'est une phrase c'est même une question qu'il pose à quelqu'un «Est-ce qu'on est sorti du monde? » Est-ce qu'on est sorti de la crise ? Tout le blabla... Mais cette phrase là qui n'a rien d'une citation, pour moi ça a une valeur incroyable mais je ne la mets pas en comparaison avec les citations. C'est pour dire qu'une phrase de Sony peut complètement m'habiter, mais pourtant ce n'est pas une phrase qui est toute proverbiale, avec des guillemets. C'est une question qu'il pose mais qui était juste une question qui est complètement dans un dialogue, de manière terre à terre. Mais comment moi dans ma tête, cette question là qui était une question terre à terre, moi dans ma tête ce que ça provoque en moi, c'est que je ne m'arrête pas au fait qu'il a posé une question à Riforoni, moi ça me pose d'autres questions à moi... Est-ce qu'aujourd'hui on est sorti de la gabegie? Est-ce qu'on est sorti de la léthargie? Quand je joue Mantsina et que je dis « Sommes-nous sortis du monde? " C'est que moi je déplace cette phrase là du contexte dans lequel Sony le met dans « Antoine m'a vendu son destin », j'enlève le contexte, que c'est Antoine qui le dit et qui dit à Riforoni «Et-ce que le peuple est prêt pour qu'on parle ? Est-ce que le jour s'est levé ?» Je l'enlève du contexte de cette pièce-là. Je prends juste cette phrase-là. Je la décontextualise complètement, et cette phrase-là elle tient en ellemême. Même enlevée de son contexte de la pièce et de sa situation théâtrale. 
Quelle trouvaille vous a fait jubiler chez Sony?

« Trouvaille » c'est un peu ce que j'ai dit au début, c'est arriver à me faire dire ou à m'apprendre de manière implicite, que la parole est une action et qu'elle s'exprime en étant une seule chose, qui ait son fond et sa forme, et que le problème de la forme doit être évidemment la nappe, la couche, l'odeur réelle de ce qu'on a à raconter, ou de ce qu'on a à dire, ou de ce qu'on a à présenter. Cette forme même de la présentation de la chose, cette forme même de la présentation du langage est quelque chose qui ne doit pas être empruntée mais qui doit naître de son « pourquoi » et son « comment ». Et que c'est la plus belle manière pour moi que Sony m'a appris, pour moi Sony par là il m'a appris la plus belle manière d'être au monde. C'est la plus belle manière d'être au monde.

Qu'est-ce qui dans sa démarche vous inspire en tant qu'artiste?

Laquelle de démarches ? II y a en a eu plusieurs. Je ne sais pas si c'est en tant qu'auteur, en tant directeur de troupe de théâtre, en tant qu'homme politique... La démarche de Sony, il a des démarches. Même si toutes ces démarches sont une seule chose à l'intérieur de la personne, c'est quand on parle de la personne on voit la personne Sony, donc évidemment il a une démarche qui s'explique comme tout le monde, en beaucoup de diverses expressions. Moi je crois que le fait que Sony ait écrit et qu'il ait mis en scène et qu'il ait parlé et qu'il ait enseigné, je trouve que c'est un «super complet ». C'est « super complet » avec chaussures chemise veste et pantalon et chapeau, tu vois non? Je veux dire ça fait pas il portait un pull, puis après en bas il est à poil et il porte des chaussures non, non. II a le pantalon, les chaussures, le T.shirt... C'est le «complet ». Et je crois que c'est cette chose là, pas que j'ai voulu être, mais que de toutes façons je ne pouvais que être, même si je n'avais pas le «complet Sony », mais que, évidemment par la rencontre de Sony, il m'a beaucoup plus réconforté à cet endroit-là. C'est que le mec il était auteur, metteur en scène, comédien, enseignant, parleur, tout le blablabla. Ça ne veut pas dire que parce qu'il accumulait toutes ces choses-là, il avait une valeur... Non, non. On peut faire une seule chose et être un Dieu comme Shakespeare, le problème n'est pas là. Ce que je veux dire quand je parle du « complet » de Sony qu'il avait tout ça en étant complet, c'est pas en terme de «il fait ça puis il a fait ça donc il est complet » c'est en terme de "parce qu'il faisait une seule chose ». C'est que cette chose-là dans sa quête, fallait qu'elle s'exprime et qu'elle s'entende. C'était une seule chose qui était « poète » c'est tout. Et c'est parce qu'il était poète qu'il a eu besoin du plateau pour qu'on entende le poète. C'est parce qu'il était poète qu'il a eu besoin d'enseigner. C'est parce qu'il était poète qu'il prenait même la parole dans les médias qu'il écrivait dans le journal la semaine pour dire «lettre ouverte au président de la République », «lettre ouverte à la France», pour dire, pour dire, pour dire. Qu'il a pris position pendant la Conférence Nationale à Brazzaville, pour la loi et la démocratie. Qu'il a parlé à la Conférence Nationale. Qu'il a pris position pour l'avènement de la démocratie à l'endroit purement politique. Parce qu'évidemment il est poète. Donc évidemment il ne fait pas tout ça pour dire que «tiens j'ai un boulot maintenant je vais apprendre ceci », comme on fait «je sais faire ça mais maintenant je vais prendre tel cours pour aussi apprendre ça et devenir ça... » Non, non. Je ne crois pas qu'il apprenait la mise en scène, je ne crois pas qu'il apprenait l'enseignement... Je ne crois pas qu'il dirigeait les comédiens puis après il dit «bon, j'ai dirigé les comédiens c'est une chose maintenant je vais apprendre à devenir enseignant pour aller enseigner les langues ». Je crois que c'est une seule chose qu'il faisait et qu'il utilisait tous ces différents canaux-là. C'est des canaux qu'il utilisait pour faire entendre la parole du poète, c'est tout. II utilisait ces différents canaux donc il n'apprenait pas autre chose. Donc c'est une parole qui sommeille dans son bide et il se bat à vouloir la sortir. Et il y a une urgence. Et comme il y a une urgence il faut qu'il la sorte et maintenant. Et donc du coup il va utiliser tous les canaux possibles qu'il aura entre ses mains pour faire entendre cette parole-là. Et si les canaux on ne les lui donne pas il les invente. Si on ne les lui donne pas il les invente. Moi je crois que si Sony n'était pas mort, il allait même être réalisateur. II allait même un jour prendre la caméra et réaliser le film. Ça ne serait même pas pour devenir réalisateur, ça serait pour faire entendre quelque chose qu'il a envie de dire. S'il se 
rend compte qu'actuellement avec l'audience, le théâtre est restreint à l'endroit de l'audience, alors il allait tourner un film. Mais pas parce qu'il doute du théâtre mais parce qu'il a besoin que ce soit entendu, genre par le plus large des gens, à ce moment là je vais faire un film. Et il allait faire un film avec La vie et demie ou Machin la hernie, il allait le faire mais pas pour être réalisateur. Donc c'est un désir qui vient réellement de comment accoucher quelque chose ce qui sommeille en soi, et une fois que tu l'as accouchée, comment faire évidemment pour arriver à son accomplissement. Parce que le fait de la pensée n'est encore que la matrice de dire que «j'ai pensé ». Le fait de l'écrire n'est que les premiers pas de dire que «j'ai pensé ». C'est à dire ça s'accomplit par une action qu'on va mener. Donc de la pensée, de la gestation, à la formation de la pensée, à son écriture, à monter sur un plateau, à le donner au spectateur puis après distraire les spectateurs, puis à enseigner à ses élèves, ce n'est que la continuation de cette affaire. C'est la continuation jusqu'à ce qu'elle s'accomplisse. Donc c'est complètement un seul canal qui continue comme ça. C'est une chose que j'ai trouvée très forte, parce qu'il ne pouvait pas s'arrêter à dire que «j'ai écrit, j'ai fait mon boulot », parce qu'il n'avait pas de boulot justement. Mais si le gars avait considéré l'écriture comme étant un boulot il pouvait dire «j'ai écrit j'ai fait mon boulot. Maintenant que les metteurs en scène fasse leur boulot, maintenant que les comédiens fassent leur boulot, maintenant que les enseignants fassent leur boulot, maintenant que les maîtres conférenciers fassent leur boulot », non, non, non. Évidemment comme ce n'était pas un boulot pour lui écrire, donc du coup il ne pouvait pas s'arrêter à écrire, il ne pouvait pas dire «j'écris c'est fini »non. Comme évidemment c'est une espèce de sacerdoce réel qu'il a à l'intérieur de lui, de quelque chose qui doit témoigner, c'était même pas l'écriture en somme qui l'intéressait, il ne faisait pas l'apologie de l'écriture. De dire qu'un auteur c'est génial, qu'il faut écrire, c'est noble et intéressant, non, non, non. C'est quelqu'un qui a quelque chose à dire. Et il trouve ce canal là. Donc c'est pas l'écriture qui l'intéressait en somme. Donc c'est pour ça que ce canal là même quand il est dedans il peut pas s'y asseoir, parce que ce n'est pas le canal qui l'intéresse. II ne peut pas s'y asseoir. Donc c'est ce que moi j'appelle le "complet». Chemise pantalon et veste. Mais ce «complet » il n'est complet que parce que lui la personne est d'abord intègre envers sa mission à lui de dire que j'ai envie que le gamin là aille à l'école, j'ai envie que la maman là... pas que la maman vienne au théâtre, c'est pas ça son problème... Que la maman sache ou apprenne ceci. Sache dans quel état honteux on est dans ce pays, on est dans ce monde, on est dans cette Afrique, on est dans ces rapports mondiaux là. C'est ça sa question. La question n'est pas que le théâtre soit beau. Et donc le théâtre devient un endroit comme un autre de dire aux gens dans quel état on est. II n'était pas là pour fêter le théâtre. Ni pour célébrer le théâtre. C'est pas l'art en somme qui l'intéresse. L'art est un canal. Et donc quand il est dans ce canal là, il ne s'assoit pas dans ce canal là, parce que son problème c'est pas le canal. Le canal c'est pour le conduit, c'est pour drainer l'eau, pour ça aille dans la pompe du voisin pour que ce voisin là ait l'eau quand il va ouvrir son robinet. Donc c'est ça la démarche qui m'a beaucoup intéressée chez Sony. C'est cet endroit là qui m'a appris que tu ne dois pas t'installer dans la forme, tu ne dois pas t'installer dans la fonction, parce qu'on est pas là pour la fonction justement. La fonction n'est qu'un canal.

Dessine-moi un Sony

Je crois qu'il ne fallait pas qu'il soit dessinateur. Parce qu'il y a un dessin de Sony que j'adore qui est au début je crois dans le bouquin "l'Acte de respirer ", ou il dessine une espèce de tête comme ça, avec un bec là tout le blablabla. Le défaut c'est qu'il était dessinateur et pour moi c'est une espèce de Picasso, parce qu'il regrettait il disait même « Ah si j'étais Picasso... ». Je crois qu'il était un vrai vrai dessinateur, c'est à dire il ne pouvait que dessiner que, quand je dis comme Picasso c'est à dire je peux aussi dessiner comme un autre, mais c'est à dire par cette lecture réelle, il dessine l'âme de la chose, mais pas l'âme dans sa pureté et pas non plus l'âme dans son côté salasse ou vilain mais c'est à dire l'âme, la chose qu'on ne peut pas dessiner. Donc du coup, on ne lui prête que des formes. Mais ces formes-là ne sont pas des formes dessinables, ni des formes écrites, ce sont des formes de sensations, de ressentis. C'est 
à dire, je vais dessiner la haine, la haine n'a pas un dessin genre carré, rond ou triangle, quand tu fais ce dessin là c'est la haine, non. La haine n'a pas de dessin. Parce que c'est un sentiment, c'est une sensation, c'est un truc qui n'a pas de forme. Donc dessiner complètement comme ça comme il le faisait, c'est évidemment la littérature de l'âme. C'est le dessin de l'enfant où il ne dessine pas la forme ; il croit qu'il dessine la forme, mais l'enfant veut absolument dessiner les sentiments. Il veut dessiner comment il aime ce bonhomme-là. Et du coup ça ne marche pas proportionnellement parce que la forme ne veut pas des proportions. L'enfant il veut qu'on sache qu'il aime le petit bonhomme de neige. Donc il veut que dans son dessin on sache qu'il aime le petit bonhomme de neige. C'est ça qui veut dessiner. Il ne veut pas dessiner comment le petit bonhomme de neige est, il veut dessiner comment lui, il aime le petit bonhomme de neige ; ou ce qu'il déteste chez Diabolo et Pénélope... Le défaut c'est que lui-même il a dessiné, et quand j'ai vu ce dessin je me suis dit «Ah merde, il m'a piqué comment moi j'allais le dessiner ». Parce que j'allais le dessiner comme ça, cette tête-là, avec des piques sur la tête, et un bec comme ça, et un œil là et puis l'autre, il n'y a pas d'œil... Oui parce que c'est la beauté du monstre, Sony c'est la beauté du monstre. Ce n'est pas le monstre beau, c'est pas le monstre joli, c'est la beauté du monstre. Et là beauté du monstre évidemment n'est pas que le monstre soit dégueulasse, n'est pas que le monstre évidemment soit gentil, la beauté du monstre c'est une chose qui est apparemment dépouillée d'un certain nombre de sentiments collés ou figés. Il n'est qu'une forme d'existence réelle. Comme quelque chose d'entier et qui ne peut pas se négocier et qui ne peut pas négocier, qui ne peut pas troquer, tu ne peux pas identifier à un autre, c'est pour ça que c'est un monstre. Nous, on est des êtres humains on se ressemble, mais un monstre est toujours spécial. Tu as un monstre et puis un autre monstre et ils n'ont pas de famille. Un monstre c'est un monstre. Sony c'est une espèce de chose toute seule comme ça, entière. C'est là où il est un monstre. Parce que c'est un monstre. Donc c'est pas beau, c'est pas laid, ça ne fait pas partie de nos vocabulaires, ça ne fait pas partie de nos trucs à nous, de juger, de regarder, d'équilibrer, tout le blabla. Mais il est, par une opération que lui même avait fait intrinsèquement, qui est une opération de devenir. C'est à dire de s'arroger une place. Il s'arroge une place pour refuser l'assignation. Il ne veut pas être assigné. Il ne veut pas qu'on l'appelle " poète engagé », il ne veut pas qu'on l'appelle "poète ». Il ne veut pas qu'on l'appelle "écrivain ", il ne veut pas qu'on l'appelle "metteur en scène ». Il ne veut pas qu'on l'appelle, il veut que lui s'appelle. C'est en ça déjà qu'il crée le monstre en lui. Et c'est une technique de dissuasion assez importante. Pourquoi ? Parce qu'à ce moment là, il crée une place. Il crée une place et il est vu. C'est à dire, il est entendu. Quand je dis qu' «il est vu il est entendu » c'est pas que sa question c'est pour qu'il soit vu, la question c'est pour que ce qu'il dit soit entendu. Or ce qu'il dit pour que ce soit entendu, il faut que ce soit lui qui le dise. Donc ça veut dire quoi ? Il faut qu'on l'entende lui pour qu'on entende ce qu'il dit. Et du coup, par là il crée une mise en scène. Et la mise en scène il la fait où ? À l'intérieur de son propre corps. Il crée une mise en scène, c'est à dire il s'arroge une place. En commençant par refuser l'assignation. Et en refusant de dire «on m'appelle». Il dit «je m'appelle». Et il le fait il dit: «je m'appelle Sony Labou Tansi ». Sur le papier c'est écrit Sony Marcel, mais il dit: «je m'appelle Sony Labou Tansi ». Et tout le monde sait par quelle étape il est arrivé pour arriver à Sony Labou Tansi, c'est même ce qui prouve qu'il s'est fabriqué. Il a étudié beaucoup de pseudonymes avant : Marcel Malinda, Soyi Soyinka... Il a eu beaucoup de pseudonymes comme ça, même les 
premiers textes il les a publiés avec d'autres pseudonymes. Mais c'est pas qu'il se cachait derrière ces pseudonymes-là, il était en train de se chercher. Et un jour quand il a trouvé Sony Labou Tansi, là c'était le monstre : le monstre, le bec était là, l'oreille était là et au-dessus de la tête il y avait des ailes de dragon et au niveau des pieds qui étaient chétifs comme ça il y a avait des crocs, le monstre était complet. Et le monstre était complet comme quand il a trouvé le nom Sony Labou Tansi. Et du coup, rien que par cette recherche de nom, et c'était pas un nom qu'il cherchait c'était une forme d'identité réelle. Et cette identité réelle il ne peut pas l'emprunter. Il ne peut la trouver qu'à l'intérieur de lui. Donc il faut qu'il rentre, et dans ses goûts, et dans ses cauchemars, et dans ses fantasmes, et dans ses colères, mais du soi, c'est à l'intérieur de lui qu'il va réveiller tout ça. C'est à dire il n'a pas peur de regarder la vérité en face. "Regarder la vérité en face " veut dire quoi? Regarder l'horrible aussi. Veut dire quoi ? Regarder la beauté aussi. Il va tout te regarder. Tu vas chier il va regarder ton caca pour savoir dans quoi on est. Quelqu'un va être content il va regarder son sourire, il est content. Quelqu'un va tirer sur quelqu'un la tête explose, le cerveau gicle il va pas faire «Oh je vais pas regarder ». Il va regarder, réellement, pour dire "voilà la merde que nous sommes, voilà les conneries que nous faisons", il va regarder. Donc il n'y aura aucun endroit où il va chercher à jouer d'une complaisance donnée parce que sinon il va corrompre la pensée, et donc du coup, l'acte de témoigner du monde par l'homme. C'est ça évidemment le monstre. C'est ça la beauté du monstre. C'est qu'il va regarder ça. Il va complètement regarder ça. Il va dire «c'est moi qui regarde». (17:27) Il y a tout un travail réellement en acceptant l'homme, c'est pour ça qu'il dit que "l'être humain est trop beau pour qu'on le néglige ». En acceptant l'homme avec ses qualités et ses défauts, il faut d'abord qu'il l'accepte pour qu'il se batte à le ré-équilibrer. Pour qu'il se batte à le ré-équilibrer.

Donc voilà, Sony c'est un processus de devenir. C'est juste un processus de devenir. C'est pas un excellent auteur, c'est pas un excellent poète, c'est pas un excellent dramaturge, c'est pas un excellent metteur en scène, c'est pas un excellent professeur, c'est pas un excellent maitre conférencier, c'est un excellent sorcier. C'est à dire, comme les vieilles matrones, c'est comme le gars dans Astérix et Obélix qui fabrique la potion magique, c'est un vieux sorcier qui fabrique un filtre qui s'appelle «le processus de devenir ». Le reste, il nous embrouille en écrivant une pièce de théâtre, en écrivant un roman, en écrivant un poème, ce sont des petits montreurs qu'il utilise, qui font partie de son procédé pour que les gens deviennent. Pour raconter son évangile à lui du processus de devenir. Il n'est pas metteur en scène, il n'est pas auteur, tout ça il s'en fout éperdument. C'est pour ça qu'il ne peut pas faire une conférence sur la littérature. C'est pour ça que la littérature ne l'intéressait pas. Il n'a jamais fait de conférence sur la littérature ça ne l'intéressait pas. Quand on lui proposait ça il dit : «on ne peut pas parler littérature ça n'a aucun sens », parler de la culture ça n'a aucun sens. C'est à l'être d'avoir la culture, on doit enseigner à la personne d'avoir la culture, mais on ne peut pas enseigner la culture, la culture c'est complètement se caresser les roubignoles sous le soleil. Ça n'a aucun sens. Sony c'est juste un sorcier. C'est une grand-mère qui fabrique des filtres, qui va te les donner comme ça quand tu vas les boire, tu n'auras pas froid pendant la saison, que le serpent ne va pas te mordre quand tu rentreras dans la brousse, c'est tout. C'est tout. C'est tout. C'est un petit sorcier. C'est un sorcier. C'est un sorcier. C'est l'art du sorcier. Il va passer par la transe pour vous déloger de chez vous. Comme ça tu vas te déloger de ton toi, tu vas te déloger de toi, pour évidemment être à même de 
regarder l'horrible en face et de regarder la beauté en face. Tu ne peux pas l'être si tu ne rentres pas en état de transe, donc il va provoquer la transe aussi, il va fabriquer un filtre qui va te faire boire. Ben oui ! Tout ça là, c'est juste un sorcier. Et moi je crois que si Sony n'était pas parti à l'école, il sera toujours Sony. Contrairement à ce que beaucoup de critiques littéraires ont dit en disant « oui c'est quand même la langue française qui l'a sauvé, parce que tu vois, en étant parti à l'école... » tout le blabla « ... ça l'a amené à écrire. Si Sony n'était pas parti à l'école, on n'aurai pas eu Sony ». Moi je dis je crois pas. Je crois que si Sony n'était pas parti à l'école, il serait toujours Sony. Il sera Sony. C'est à dire il serait pas le mec qui écrit bien sûr mais ce sera toujours le même Sony. Ça veut dire quoi ? Il sera un putain de sorcier dans son village. Il allait être un sorcier. Je ne dis pas forcément un gourou qui crée une secte, pas à cet endroit là. Mais il sera un sorcier et qui sera aussi connu tel qu'il est connu en tant qu'auteur. Il n'est pas mondialement connu, il n'est pas africainement connu. C'est à dire dans le petit espace, je dirai même pas célébrité, de l'endroit où il est connu, quand je dis connu, je parle du verbe connaître, c'est à dire où l'on s'est approché de sa littérature, où on a fait l'amour avec sa littérature. Donc on le connaît comme on connaît une femme, comme un homme une femme, ils ont fait l'acte de connaissance, tu vois ? Voilà. Donc tu coup s'il n'était pas auteur, on aurait connu ce qu'il allait faire là, on l'aurait connu parce que c'est un sorcier, il est sorcier. Et sa manière de penser ne vient pas de l'école, ne vient pas parce qu'il a lu un bouquin. Ils étaient nombreux à lire ces bouquins là. Parce que intrinsèquement il y a quelque chose qui est née révoltant. Qui est née révolté. Quelque chose qui a été baigné dans la honte depuis sa naissance. Il est sorti dans cette cosmogonie où il vécu, avec ses histoires familiales, ses histoires de pouvoir. Quand il vient au monde, il arrive dans un pays où il $\mathrm{y} a$ un pouvoir comme ça, il est gamin, on mange dans la poubelle, il y a le oui et le non c'est la même chose, il y a la honte de la famille, il y a tout ça donc bébé, il nait et il se tape tout ça dans la gueule. Ok? Mais beaucoup de gens ont eu ces mêmes trucs là. Il n'était pas le seul qui est née pendant cette période là, ou qui est né dans ce village là, ou qu'on a aussi envoyé à l'école. Beaucoup de gens se sont tapé ces problèmes là. Mais pourquoi lui ça lui a fait ça? Ah ben parce que ces mêmes réactions, quand c'est tombé chez lui, ça a provoqué ceci pourquoi ? Parce que évidemment il a un cœur de beurre. Ça fond. Et donc du coup, il est complètement transparent, il est complètement transperçable. Il est complètement transperçable, il est complètement mortel. Il est complètement la peur. Le mot qu'il dit la peur et la honte. Donc tout le monde le traverse comme ça, depuis petit. Le traverse comme ça. Donc il s'en rend compte, très bien compte, qu'on lui donne un sursis de 5 ans à vivre. Il a 5 ans pour vivre à après il crève. C'est comme un prématuré mais pas dans de le sens d'être né avant, il a des insuffisances comme quelqu'un qui a des insuffisances de cœur. Le docteur a dit «toi tu as 10 ans (à vivre) », il sait qu'il a 5 ans à vivre. Alors ce qu'il va chercher c'est quoi ? C'est pas de vivre longtemps. Ce qu'il va chercher évidemment, c'est ça qui est beau, c'est que par sa maladie, il va chercher à reformer le monde. Pour dire que : « il y en a beaucoup qui ont vécu la même maladie comme moi, il y en a beaucoup qui pourront encore vivre cette maladie-là, il y en a beaucoup qui pourront vivre ces carences là. Donc il faut que je me batte pour que les enfants de demain ne souffrent pas de cette carence là. » Mais il n'a pas le médicament, alors qu'est-ce qu'il fait? Il s'invente docteur. Dans le film documentaire "Diogène à Brazzaville » ça s'explique, quand il arrive à l'école à Brazzaville, il quitte la RDC, donc l'ex-Congo Belge à l'époque. Quand il arrive au Congo, dans la salle de classe, il 
se mettait à prendre des récréations, il se mettait toujours au coin de la classe, en train de créer des pénicillines. Lui même il le raconte dans le film "Diogène à Brazzaville ", en train de créer des pénicillines il attrape les insectes, il prend des bouts de bois, il crée des trucs comme ça et chaque fois l'instituteur le punissait pour dire «Ah ça c'est Sony qui est train de faire des fétiches pour que les autres élèves ne soient pas admis et que lui passe. Non, non, non. Il était déjà dans son laboratoire, dans son atelier. Mais pourquoi il voulait faire un atelier? Gamin à l'école primaire, pourquoi il avait besoin de faire un atelier ? C'est que c'est d'abord un sorcier, c'est un être dans un laboratoire, c'est un savant. Il croit qu'il va fabriquer une espèce de Nivaquine, qu'on doit la prendre et fini le paludisme. Mais bien sûr! Donc il a ça depuis gamin et cette pénicilline qu'il invente comme ça avec ses bouts de bois, avec je sais pas moi... du sperme de lilliputien, avec la sève de tel écorce d'arbre, ou la morve de je ne sais pas quel animal, de quelle chèvre dans le quartier qu'il a ramassée, lui il croit qu'en mélangeant ça, on inventera un truc qui arrêtera le sousdéveloppement. Donc il est comme ça avec toute l'innocence du gamin. Et quand on lui confisque son laboratoire dans la classe, il s'énerve, il a pris une feuille et il a rempli la feuille d'encre. C'est à dire, il a écrit. Ça veut dire quoi ? Il continue à faire le laboratoire maintenant avec le stylo. Donc c'est pas l'écriture qui l'a sauvé, c'est pas l'écriture qui créé Sony, c'est quand on a confisqué son laboratoire. Qu'est-ce qu'il fait? Il continue le même laboratoire par un autre outil, par un autre outil c'est tout. Il continue son laboratoire avec le stylo. Et voilà pourquoi il est réellement poète à cet endroit d'être poète parce qu'il n'est pas poète par le papier ni par le stylo ni par la machine à taper. Il est poète parce qu'il veut inventé un médicament. Donc si c'est pas avec le stylo il fera avec le pinceau, s'il n'a pas le pinceau il le fera avec du pain, c'est pour ça que je ne crois pas quand les gens disent «si Sony n'était pas parti à l'école il serait perdu... » Non il serait toujours Sony. Il serait un sorcier, un magicien, un machin...

Il sait très bien qu'on ne l'a pas inventé. Et c'est pas par orgueil tapageur qu'il le dit, il le dit dans une position très très consciente d'un mec dans son laboratoire avec ses pénicillines au fond de la classe. Il n'a pas demandé « est-ce que c'est juste ?» Il n'a pas d'abord chercher la vraie Nivaquine le vrai machin et connaître le Ph non, c'est dans l'innocence complètement du fou, qu'il ne sait même pas si scientifiquement cet acide là marche avec ça non, non, c'est le courage de se dire que « je vais inventer ma science ». Donc il croit comme un gamin qui joue, comme les filles jouent avec leur poupée, les gamins qui jouent avec les petites voitures, et ces machines qui rentrent là et lui il devient le héros. Il croit à ça dans cette réelle innocence. Et c'est là que le monstre se crée. C'est là qu'on rentre déjà dans la fabrication du monstre. C'est qu'il commence à s'auto-fabriquer monstre. Monstre pourquoi ? Parce qu'à ce moment là il fait du Nietzsche «Ni Dieu ni Maitre». "Je ne vais pas dire qu'est-ce qui est vrai qu'est-ce qui est faux des autres trucs qu'ils utilisent, la vérité c'est ce que moi je vais dire ». Et c'est pour ça qu'il met encore cette phrase là dans "l'Acte de respirer ", quand il dit « le mal est mort le mal est mort le mal est mort, et le bien, le seul bien qui existe c'est moi. Il vente en moi, il bouge en moi, il fait lune en moi. » Mais quand il dit «le bien qui existe c'est moi » c'est pas qu'il est en tain de dire "c'est moi l'homme le plus beau, l'homme le plus gentil ou des leçons de moral» non, non. J'invente mon bien. J'invente le bien. J'invente. C'est j'invente, ça ne se vérifie pas. Ce que j'invente tu ne peux pas le vérifier avec autre chose. Ça ne se vérifie pas. C'est dans ma cosmogonie. Et c'est là évidemment l'écriture du monstre. C'est là 
évidemment la statue (stature ?) du monstre. C'est là où le monstre est différent de nous, parce qu'il n'y a qu'un seul monstre. Après tu as un autre monstre, ils ne se ressemblent pas, les humains se ressemblent, c'est là le monstre justement.

Un jour, il y en aura qui diront « je l'ai influencé », ils seront nombreux et voici ma réponse à tous ceux-là qui croient qu'ils m'ont influencé. Je dirai : «d'accord, vous m'avez influencé, mais je suis allé plus loin que vous, j'ai sauté plus haut que vous, accusez-moi de cela, pas d'autre chose, autrement soyez fiers de m'avoir engendré. C'est votre droit après tout. SLT.

Tu ne peux pas influencer Sony c'est impossible (référence à la citation donnée), c'est impossible. Tu peux être meilleur auteur que lui ça c'est très clair, tu peux être meilleur dramaturge que lui, tu peux être meilleur metteur en scène que lui ça c'est très clair, tu peux être meilleur enseignant que lui ça c'est très clair, tu peux être meilleur tout ce que tu veux, tu peux être meilleur formateur que lui ça c'est très clair, mais tu peux pas être meilleur que Sony ça c'est impossible. Et ça, ça n'a rien à voir avec le fait qu'il écrive. Ça n'a qu'à voir avec l'organe pensant qu'il est. Cette espèce de macro-tracteur. Avec des doigts de chair quand on dormait là le lendemain il a fait des tentacules, cette espèce de macro-tracteur sorti de la terre. Voilà. Et c'est pour ça que sa première pièce s'intitule "Conscience de tracteur ». C'est la première pièce de théâtre qu'il écrit, il publie en 1976. Et le début de cette pièce dit ceci, c'est aussi avec ça que Ferdinand avec Léandre- Alain Baker quand il fait le film à Brazzaville, c'est le premier texte que tu entends dans le film, voix off tu entends cette phrase là, après on montre le fleuve Congo, une pirogue qui passe, et surtout cette voix, cet extrait-là dit comme ça en voix off par Pascal Nzonzi avec une meilleure voix où il dit dans le prologue de Conscience de tracteur où il dit: «la première terre est passée, la deuxième terre est passée, la troisième terre est passée, la quatrième terre est passée, la cinquième terre est passée, il y eu 550 millions de terres passées, 550 millions de terres passées emportées à la dérive par l'espace et le temps. Puis le hasard en se mouchant fit l'homme. Et donc, si le hasard ne s'était pas mouché... Il lui donna deux bras et deux mains pour manœuvrer des mondes, de telle sorte que toute la création ne soit qu'une éternelle ébauche. Ébauche ou débauche? C'est à voir. » C'est comme ça qu'il commence «c'est à voir ». Mais le gars, c'est pas de la littérature. C'est de la philosophie pure. Mais laquelle? Mais pas la philosophie de la philosophie. C'est le monstre qui est train de créer son laboratoire. Le monde est inachevé et je crée le mien à l'intérieur. Et ça c'est ce qu'il a fait dans toutes ses œuvres, romans, poèmes, nouvelles, pièces de théâtre, par les premières phrases. Les premières phrases il te dit "c'est foutu ", il dit « la première terre est passée ». Dans "Antoine m'a vendu son destin » il commence comment? «Sommes-nous sortis du monde, Riforoni ?", dans "La parenthèse de sang", il commence comment? Il dit "Ça commence ». Lui il prend le stylo, il le pose sur la feuille, il dit «ça commence " mais c'est pas moi qui commence à écrire, le monde commence, à partir de maintenant. Comme dit Shakespeare au début de Richard 3 « Et voici l'hiver de notre déplaisir », « et maintenant ", ça commence par moi, par l'acte de nommer et que moi je crains. Il dit ça commence, ça commence comme un match de football mais ça ne termine pas. "Les onze du sang contre les onze des entrailles. Et pas d'Afrique dans ce match s'il vous plait, car la situation de l'Afrique est à la limite distrayante. Attention l'arbitre est un ancien fou, il siffle à l'envers. » L'arbitre tu sais c'est qui ? C'est lui. "Attention l'arbitre est un ancien fou, il siffle à l'envers. " Donc cette littérature là, c'est à l'envers. Ne la jugez pas par rapport à comment vous lisez le 
monde, vous allez vous tromper. Retournez là. Retournez là complètement. "L'arbitre est un ancien fou, il siffle à l'envers. " Comment il commence La vie et demie? « Tout avait commencé quand Chaïdana avait eu 15 ans. » TOUT. Il dit TOUT. Pas cette histoire que je vous raconte. Le monde. Avec les Abraham qui avaient existé avant les Mama Ngounga, les Soundiata Keita, les Napoléon, tout ça avait commencé quand Chaïdana avait eu 15 ans. Pour le comprendre tu peux dire "non c'est peutêtre moi qui exagère, c'est peut-être pas ça, mais lis la deuxième fois tu vas comprendre." Il dit "Tout avait commencé quand Chaïdana avait eu 15 ans.» Deuxième phrase : « Mais le temps est par terre ». Par terre, par terre. L'air par terre. Il cite tout, par terre. Il dit "même la terre aussi était par terre ». Quand Chaïdana avait eu 15 ans, tout est par terre. C'est à dire, c'est le Big Bang. Sony nous parle du phénomène du Bing Bang. Dans toutes ses œuvres. Dont son dernier roman «Le commencement des douleurs", publié de son vivant je veux dire. Ça commence comment? Il dit « tout avait commencé par un baiser puant. Baiser de malheur. Nous avons eu tort, nous à qui l'histoire a piqué 5 siècles.» Il commence comme ça. «Dans les yeux du volcan ", comment il commence ? Il dit "Cétait le vendredi de machin, quand le colosse arriva sur son cheval dans la ville des crânes... » ben oui, c'est là que c'est parti « quand le colosse a débarqué sur son cheval dans la ville des crânes tout était foutu », comme quand je dis dans « Le socle des vertiges » en disant «tout avait mal commencé quand Diego Kawa a débarqué de son bateau c'était foutu ». Ne cherche pas d'explication c'est foutu. Fallait pas qu'il débarque du haut de son bateau. Dès qu'il a débarqué du haut de son bateau c'était foutu. A partir de là on ne peut plus rien comprendre. Les choses sont devenues sens dessus dessous. Il n'y a plus à expliquer mais c'est mal parti comment est-ce qu'il y a un endroit qu'on a pas bien compris... Non! C'est quand le bateau de Diego Kawa a fait POUM, il n'y a aura plus d'explication, il n'y aura plus rien. Toutes les œuvres de Sony, tu l'as depuis le début. Tu comprends très bien que le gars il est dans son laboratoire, il recrée le monde. À partir de son laboratoire. Et sur l'histoire de l'envers, de l'arbitre qui siffle à l'envers, il le dit dans la préface de « La vie et demi », il dit dans la préface « la vie et demi ça s'appelle écrire par étourderie. Moi qui vous parle de l'absurdité de l'absurde, d'où voulez-vous que je vous parle sinon du dedans de moi-même ? ». Du dedans. Il crée son laboratoire à l'intérieur de ses couilles, de ses tripes, il se crée sa petite maison à l'intérieur, il invente ses pénicillines. Et donc c'est par étourderie qu'on doit le comprendre. Parce que c'est un ancien fou. Il siffle à l'envers. Là où il ne faut pas siffler pénalty, il siffle pénalty. Quand vous dites que le match est fini, il dit « Non! Le match commence maintenant». Quand vous jouez il dit «Ah non! Vous êtes sérieux ». Quand vous êtes sérieux, il dit « Ah ben non, la vous jouez ! ».

\section{RÉSUMÉS}

Entretien repris du blog du festival Mantsina sur scène. La 12e édition s'est tenue du 10 au 30 décembre 2015, à Pointe Noire et Brazzaville, Congo. Direction : Dieudonné Niangouna et Sylvie 
Dyclo Pomos

https://festivalmantsina.wordpress.com/2015/12/30/dessine-moi-un-sony/

INDEX

Mots-clés : Sony Labou Tansi, Congo, Dieudonné Niaougouna, Festival Mantsina sur scène 